\title{
Treatment Outcomes of Vagus Nerve Stimulation in Lennox-Gastaut Syndrome
}

\author{
Bu Seon Kang ${ }^{1}$ Yu Seung Woo, MD², Jinsun Lee, MD², Yoon Young Yi, MD², Bon Seok Koo, PhD², \\ Joon Won Kang, PhD $D^{2,4}$ \\ 'Department of Medicine, Chungnam National University College of Medicine, Daejeon, Korea \\ ${ }^{2}$ Department of Pediatrics, Chungnam National University Hospital, Daejeon, Korea \\ ${ }^{3}$ Department of Otorhinolaryngology-Head and Neck Surgery, Chungnam National University Hospital, Chungnam National University \\ College of Medicine, Daejeon, Korea \\ ${ }^{4}$ Department of Pediatrics, Brain Research Institute, Chungnam National University College of Medicine, Daejeon, Korea
}

Received: June 18, 2019

Revised: July 30, 2019

Accepted: July 31, 2019

Corresponding author:

Joon Won Kang, PhD

Department of Pediatrics,

Chungnam National University

Hospital, Chungnam National

University College of Medicine, 282

Munhwa-ro, Jung-gu, Daejeon

35015, Korea

Tel: $+82-42-280-8244$

Fax: +82-42-255-3158

E-mail: childlove@cnu.ac.kr
Purpose: Lennox-Gastaut syndrome (LGS) is one of the most severe epileptic encephalopathies and frequently patients with this syndrome respond poorly to antiepileptic drugs. The aim of this study was to evaluate the treatment outcomes of vagus nerve stimulation (VNS) in LGS patients with drug-resistant epilepsy.

Methods: We analyzed the clinical files, collected over 5 years, of children with LGS who received VNS treatment.

Results: Seven children were included in this study (four males, three females; mean age of VNS insertion $12.4 \pm 3.5$ years). All the patients had generalized tonic seizures and there were various other seizure types including myoclonic seizures, atonic seizures, and atypical absence seizures. Although two patients had normal imaging, five patients had abnormalities on imaging, including pachygyria, cortical dysplasia, kernicterus, and a chromosomal anomaly. Comparing the baseline seizure frequency to the frequency after the VNS surgery, the seizure frequency at the last follow-up showed a decrease of $57.2 \%(0 \%$ to $100 \%)$ on average $(P=0.028)$ and one patient achieved seizure free status. Only two children were given additional antiepileptic drugs with the aim of managing their seizures. There was no mortality or complications related to the VNS therapy except one case requiring intensive care unit admission due to pneumonia. Comparing the results before and after VNS surgery, the VNS therapy also had a tendency to have a positive effect on quality of life $(P=0.066)$.

Conclusion: In LGS patients with drug resistant epilepsy who are not candidates for a corpus callosotomy or resective surgery, VNS could be an effective, low-risk adjunct therapy for decreasing seizure frequency.

Keywords: Vagus nerve stimulation; Lennox Gastaut syndrome; Drug resistant epilepsy; Seizures; Quality of life

Copyright@ 2019 Korean Child Neurology Society

This is an Open Access article distributed under the terms of the Creative Commons Attribution Non-Commercial License (http://creativecommons.org/licenses/by-nc/4.0/) which permits unrestricted non-commercial use, distribution, and reproduction in any medium, provided the original work is properly cited. 


\section{Introduction}

Lennox-Gastaut syndrome (LGS) is one of the most severe epileptic encephalopathies of childhood onset and accounts for approximately $3 \%$ to $10 \%$ of all childhood epilepsies $[1,2]$. The sex ratio of patients with LGS is reported as half and half [3], and there is a classic triad that defines LGS. First, there are specific electroencephalographic (EEG) patterns consisting of bursts of diffuse slow spike-and-wave complexes less than 2.5 cycles per second in awake patients and generalized paroxysmal fast activity of 10 to $20 \mathrm{~Hz}$ mainly during non-rapid eye movement sleep [1]. Second, LGS patients have diverse seizure types such as tonic seizures, atypical absence seizures and drop attacks including head drop or whole body drop [2]. The third feature of LGS is impaired mental function with or without behavioral problems [3].

LGS is regarded as one of the most challenging epilepsies to manage, due to a range of different seizure types which are mostly refractory to antiepileptic drugs (AEDs) [4]. The treatments for LGS can be classified into medication, diet and surgery. The AEDs such as valproic acid, clobazam, topiramate, levetiracetam, lamotrigine and rufinamide are considered the preferred AEDs, but choosing the most effective AED is challenging and sometimes, based on patient response, a combination of drugs is necessary [3]. Recently, non-pharmacological treatment options have been administered to LGS patients including ketogenic diets, vagus nerve stimulation (VNS), corpus callosotomy and resective surgery. However, most patients with LGS cannot undergo resective surgery due to the multifocal characters of LGS or the difficulty in localizing a single seizure focus [5]. VNS is increasingly being utilized as an adjunctive therapy for patients with drug-resistant epilepsy who are not suitable for resective surgery [6]. The objective of our study was to examine the efficacy of VNS in LGS patients with drug-resistant epilepsy from a single center in South Korea.

\section{Materials and Methods}

\section{Study population and data source}

Between January 2012 and May 2019, a total of 45 patients who met all the criteria of LGS visited the Department of Pediatrics at Chungnam National University Hospital [2,3]. Among them, seven of these patients (15.6\%; four males, three females) who had drug-resistant epilepsy were treated with VNS (Table 1). The first eligibility criterion for the study population was that the age at which the VNS device was implanted was younger than 18 years. The second criterion was that medical records had to contain a minimum of 3 months of data on the patient's seizure types, frequency, and severity prior to implantation and a minimum of 24 months of follow-up data following implantation.

In addition to demographic characteristics of the patients, some features, such as the patient age at the initial visit and during the follow-up period, were obtained retrospectively from the medical records. The clinical data were also collected retrospectively from the medical records and included the age at seizure onset, age at implant, latent period until surgery, post operation follow-up period, previous history of infantile spasms and diet therapy, seizure types, cognition, and etiology.

The study focused on assessing the change in seizure frequency of the predominant seizure type from baseline, meaning the 3-month period prior to implantation, to 24 months following implantation. The predominant seizure type was defined as the most disabling seizure type noted in the medical records, not necessarily the most frequent seizure type. This study utilized a sixpoint seizure frequency scale [7], and a therapy responder was classified as a $\geq 50 \%$ reduction in baseline seizure frequency of the predominant seizure type following surgery. A six-point sei-

Table 1. Patients demographical and clinical profiles

\begin{tabular}{lc}
\hline Variable & Value \\
\hline Gender, male/female & $4(57.1): 3(42.9)$ \\
Age at seizure onset (yr) & $3.5 \pm 4.0(0.0-12.0)$ \\
$<1$ & $3(42.9)$ \\
$3-6$ & $3(42.9)$ \\
$>6$ & $1(14.2)$ \\
Age at surgery (yr) & $12.4 \pm 3.5(5.3-16.5)$ \\
$<6$ & $1(14.2)$ \\
$>10-16$ & $5(71.6)$ \\
$>16$ & $1(14.2)$ \\
Latent period of surgery (yr) & $9.0 \pm 5.2(1.7-14.9)$ \\
Postoperation follow-up period (mo) & $38.8 \pm 6.4(29.7-49.7)$ \\
Seizure types & \\
Generalized tonic & $7(100.0)$ \\
Myoclonic & $5(71.4)$ \\
Atonic & $4(57.1)$ \\
Atypical absence & $1(14.2)$ \\
Etiology & \\
Symptomatic & $5(71.4)$ \\
Pachygyria & $2(28.6)$ \\
Diffuse cortical dysplasia & $1(14.3)$ \\
Kernicterus & $1(14.3)$ \\
Chromosome abnormality & $1(14.3)$ \\
Unknown & $3(42.9)$ \\
Previous history of infantile spasms & $2(28.6)$ \\
\hline
\end{tabular}

Values are presented as number (\%) or mean \pm standard deviation (range). 
zure frequency scale recorded the clinician's assessment of the patient seizures as "seizures increased," " $\leq 24 \%$ seizure reduction or no change in seizure frequency," " $25 \%$ to $49 \%$ seizure reduction," " $\geq 50 \%$ to $90 \%$ seizure reduction," "no seizures reported," and "unknown." The safety of VNS was monitored by assessing the incidence of all adverse events (AEs) starting from the day of implantation. Clinicians' assessments of health outcomes were made using the Clinical Global Impression of Improvement (CGI-I) rating scale.

\section{VNS insertion and parameters}

VNS (VNS pulse ${ }^{\circledast} 102 \cdot 103$, Cyberonics Inc., Houston, TX, USA) insertion was performed by otolaryngologists under general anesthesia and took approximately 2 hours and was done following standard surgical procedure [8]. The procedure includes attaching electrodes to the vagus nerve in the left cervical region, connecting the bipolar lead of the electrodes to a small generator and implanting it under the skin of the left chest region. After a recovery period, the stimulation values were programmed during the outpatient visit. For all the patients, the stimulation frequency was set as $30 \mathrm{~Hz}$ with a pulse width of $500 \mu \mathrm{s}$. However, a few parameters were adjusted in each patient once the possibility of complications or changes in the seizure frequency were factored in. The stimulation strength was set to $1.5 \mathrm{~mA}$ for one patient, $2.0 \mathrm{~mA}$ for five patients, and $2.25 \mathrm{~mA}$ for one patient. The non-stimulating time after 30 seconds of stimulation was adjusted as 3 minutes for three patients and 5 minutes for four patients, and this pattern was programmed to repeat every 24 hours.

\section{Statistical analysis}

The statistical analysis was done by SPSS version 17.0 (SPSS Inc., Chicago, IL, USA). The Wilcoxon Rank Sum test was applied to the data to compare non-parametric variables and a $P$-value of $<0.05$ was regarded as statistically significant.

\section{Ethics}

This study protocol was reviewed and approved by the Institutional Review Board of Chungnam National University Hospital (2017-01-041). Due to its retrospective nature, the study was exempted from requiring informed consent from the participants.

\section{Results}

\section{Demographic and clinical characteristics of the study patients}

A total of seven patients (four males, three females) with a VNS therapy device implanted were included in this study. The average patient age at seizure onset was $3.5 \pm 4.0$ years (range, 0.0 to 12.0 ). Fig. 1 shows that three patients were younger than 1 year, three patients were 3 to 6 years and only one patient was older than 6 years. The average age at surgery was $12.4 \pm 3.5$ years (range, 5.3 to 16.5 ), one patient was younger than 1 year, five patients were 10 to 16 years and the other one patient was older than 16 years. Period until surgery was $9.0 \pm 5.2$ years (range, 1.7 to 14.9 ) and the postoperative follow-up period was $38.8 \pm 6.4$ months (range, 29.7 to 49.7 ).

In our study patients had more than one seizure type, so there are a number of findings that overlap in Table 2. All the patients had generalized tonic seizures and there were five with myoclonic seizures, four with atonic seizures, and one with atypical absence seizures. Three patients had normal imaging, while four pa-

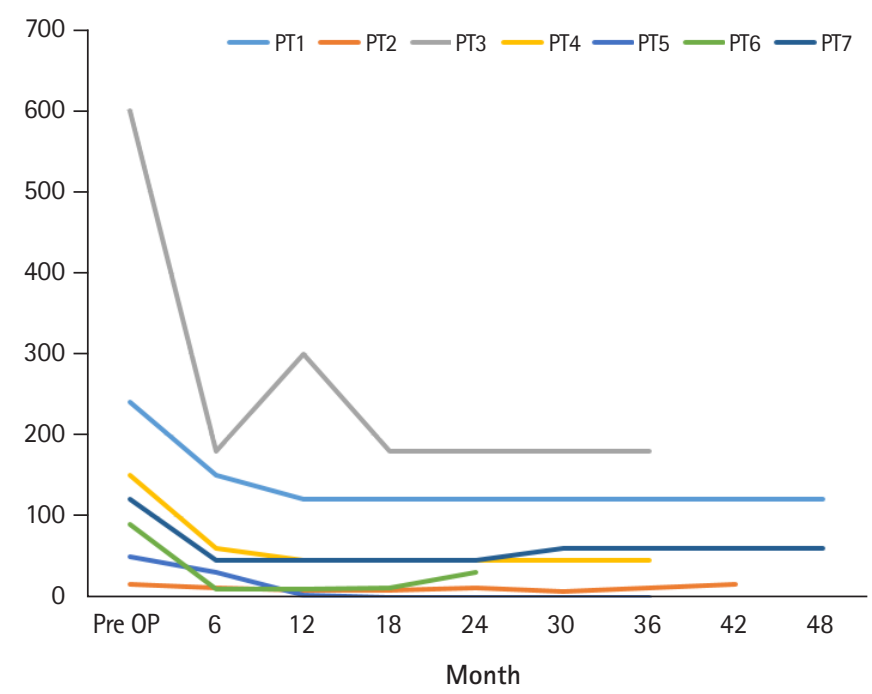

Fig. 1. Seizure frequency before vagus nerve stimulation operation $(\mathrm{OP})$, at $6,12,18,24,30,36,42$, and 48 months after the operation. The frequency was reported by caregivers at the outpatient pediatrics clinic of Chungnam National University Hospital. PT, patient.

Table 2. Changes on seizure frequency and numbers of AED

\begin{tabular}{lcccc}
\hline $\begin{array}{l}\text { Patient } \\
\text { no. }\end{array}$ & $\begin{array}{c}\text { Baseline seizure } \\
\text { frequency (/mo) }\end{array}$ & $\begin{array}{l}\text { Seizure frequency at follow-up (/mo) } \\
\text { last }\end{array}$ & $\begin{array}{c}\text { Baseline } \\
\text { AED no. }\end{array}$ & $\begin{array}{c}\text { AED no. at last } \\
\text { follow-up }\end{array}$ \\
\hline 1 & 240 & $120(50.0 \downarrow)$ & 5 & 5 \\
2 & 15 & $16(6.7 \uparrow)$ & 5 & 5 \\
3 & 600 & $180(70.0 \downarrow)$ & 6 & 6 \\
4 & 150 & $45(70.0 \downarrow)$ & 7 & 4 \\
5 & 50 & $0(100.0 \downarrow)$ & 4 & 4 \\
6 & 90 & $30(67.0 \downarrow)$ & 3 & 4 \\
7 & 120 & $60(50.0 \downarrow)$ & 3 & 5 \\
Total & 180.7 & $64.4(57.2 \downarrow)$ & 4.7 & 4.7 \\
\hline
\end{tabular}

Values are presented as number (\%).

AED, antiepileptic drug. 
tients showed abnormal image findings relating to etiology, including two with pachygyria, one with diffuse cortical dysplasia, and one with kernicterus. One patient had a chromosomal abnormality. Furthermore, there were two patients with a previous history of infantile spasms and three patients with a previous treatment history of diet therapy.

\section{Clinical outcomes after VNS insertion}

Table 3 shows that the mean follow-up period after VNS insertion was 38.8 months (range, 29.7 to 49.7). When compared with the baseline seizure frequency, the frequency at the last follow-up decreased by $57.2 \%$ on average. The largest reduction was $100.0 \%$ (from 50 to 0 times per month) and the smallest was $50.0 \%$ seen in two patients (from 240 to 120 and from 120 to 60 times per month), with the latter nonetheless a responder. The patient with the largest reduction of seizure frequency was seizure free during the last 12 months of follow-up. In contrast, there was one patient with a $6.7 \%$ increase in seizure frequency (from 15 to 16 times per month). The baseline and change in seizure frequency of each patient at 6-month intervals and at the last follow-up are shown in Fig. 1. Using the seizure frequency before surgery and at the last follow-up, the $P$-value was 0.028 which it is statistically significant. Looking closer, there were some fluctuations in seizure frequency during the follow-up period, but all the patients showed a $\geq 50 \%$ reduction in seizure frequency from the first postoperative visit, classifying them all as responders.

As can be seen in Tables 3 and 4, most patients took the same number of AEDs from baseline until last the follow-up visit. The exceptions were two children, where AEDs were added (from 3 to 4 and from 3 to 5 ) and one child who's AEDs were reduced (from 7 to 4). The number of AEDs were adjusted at outpatient follow-up visits based on the symptoms and overall condition of the patient. All the children with increase number of AED keep the existing drugs and started to take clobazam, and lacosamide plus vigabatrin each. Both of them got the decreased seizure frequency for at least 6 months after VNS surgery without any changes on

Table 3. Level of benefit achieved with vagus nerve stimulation therapy

\begin{tabular}{|c|c|c|c|c|}
\hline \multirow{2}{*}{ Patient no. } & \multicolumn{2}{|c|}{ Severity of illness } & \multirow{2}{*}{ CGI-I } & \multirow{2}{*}{ Efficacy index } \\
\hline & Before & After & & \\
\hline 1 & 7 & 7 & 4 & Unchanged \\
\hline 2 & 5 & 4 & 2 & Moderate \\
\hline 3 & 5 & 4 & 3 & Minimal \\
\hline 4 & 6 & 2 & 1 & Marked \\
\hline 5 & 4 & 4 & 3 & Minimal \\
\hline 6 & 4 & 4 & 3 & Minimal \\
\hline 7 & 7 & 5 & 3 & Minimal \\
\hline \multirow[t]{8}{*}{ Description of the grades } & $0=$ Not assessed & & $0=$ Not assessed & Marked \\
\hline & $1=$ Normal, not at all ill & & $1=$ Very much improved & Moderate \\
\hline & $2=$ Borderline mentally ill & & $2=$ Much improved & Minimal \\
\hline & $3=$ Mildly ill & & $3=$ Minimally improved & Unchanged or worse \\
\hline & $4=$ Moderately ill & & $4=$ No change & Not assessed \\
\hline & $5=$ Markedly ill & & $5=$ Minimally worse & \\
\hline & $6=$ Severely ill & & $6=$ Much worse & \\
\hline & $7=$ Among the most extrer & & $7=$ Very much worse & \\
\hline
\end{tabular}

CGI-I, Clinical Global Impression of Improvement.

Table 4. Retrospective studies on vagus nerve stimulation efficacy South Korea

\begin{tabular}{llcccccc}
\hline Study & \multicolumn{1}{c}{ Study population } & No. of centers & Total no. & $\begin{array}{c}\text { Patients } \\
\text { with VNS }\end{array}$ & $\begin{array}{c}\text { Patients } \\
\text { with LGS }\end{array}$ & $\begin{array}{c}\text { Follow-up } \\
\text { period }\end{array}$ & $\begin{array}{c}\text { Patients with greater } \\
\text { than 50\% reduction (\%) }\end{array}$ \\
\hline Kim et al. (2004) [20] & Intractable pediatric epilepsy & Multiple & 12 & 12 & 9 & $3-57$ mo & 67 \\
You et al. (2005) [21] & Intractable pediatric epilepsy & Single & 7 & 7 & 2 & $12-44 \mathrm{mo}$ & 71 \\
Kang et al. (2005) [22] & Intractable pediatric epilepsy & Single & 297 & 5 & 2 & $6-88 \mathrm{mo}$ & 40 \\
Yum et al. (2007) [23] & Lennox-Gastaut syndrome & Single & 79 & 7 & 7 & $5.0 \pm 3.3 \mathrm{yr}$ & 29 \\
Current study & Lennox-Gastaut syndrome & Single & 7 & 7 & 7 & $3.2 \pm 0.5 \mathrm{yr}$ & 86
\end{tabular}

VNS, vagus nerve stimulation; LGS, Lennox-Gastaut syndrome. 
the existing AEDs. A few months after VNS operation, the number of AEDs were adjusted at outpatient follow-up visits based on the symptoms and overall condition of the patient. Even with the adjustment of AEDs, there was no difference in seizure frequency of two children over 1 year after adding new drugs.

In terms of complications, one patient was admitted to the intensive care unit (ICU) because of pneumonia that developed on day 4 postsurgery which was further complicated by atelectasis and a right-sided pleural effusion. The patient stayed in ICU for 17 days receiving the necessary treatment for the pneumonia and was discharged from the hospital in good condition on day 30 postsurgery. Apart from this patient, there were no other AEs relating to the surgery, including the more common neurological complications such as a hoarse voice.

\section{Level of benefit achieved with adjunctive VNS therapy over time}

The level of benefit, relating to quality of life (QOL), gained from adjunctive VNS therapy was assessed by the physician following at least 24 months of VNS treatment. Table 4 shows the assessment of the patient's overall condition and makes a comparison of the patient's condition before and after VNS insertion. Three children experienced no change and four patients had an improvement in illness severity. Among those that saw an improvement, two children's assessments went from "markedly ill" to "moderately ill," and the most improved patient was assessed as "borderline mentally ill" where it was previously "severely ill." The remaining patient was initially assessed as "among the most extremely ill patients" (the worst possible condition before VNS insertion) and even her condition improved, assessed as "markedly ill" postoperatively, which is a two-step improvement $(P=0.066)$.

As can be seen in Table 4, when using the GCI-I scale, "very much improved" was the best result occurring in patient 4. Patient 2 placed second with a result of "much improved," followed by the "minimally improved" result seen in patients 3, 5, 6, 7 and finally a result of "no change" in patient 1 . Patient 1 also obtained the result of "unchanged" on the efficacy index, while four patients scored "minimal," one "moderate," and one "marked."

\section{Discussion}

LGS is a severe childhood-onset epileptic syndrome characterized by multiple seizure types with high frequency, mental retardation and an EEG pattern of diffuse, slow spike-wave complexes and generalized fast activity. In most cases LGS responds poorly to AEDs [5]. Previous studies have reported that only $6.7 \%$ to $13.7 \%$ of patients achieve seizure freedom with pharmacotherapy [9].
Various treatment options have recently been applied in LGS patients, including ketogenic diets, resective surgery, corpus callosotomy and VNS. However, the majority of LGS patients are not candidates for resective surgery due to the multifocal characteristics of the disease or the difficulty to localize a single seizure focus [10]. When medication fails and resective surgery is impossible, other alternative therapies are considered [8].

VNS can be offered as one of these alternative therapies. It is a type of palliative surgery that was originally approved by the Food and Drug Administration in 1997 as an adjunctive therapy for adults and adolescents who are not eligible for resective surgery [5]. VNS is one of the most common neuromodulation-based therapies available. The VNS system consists of a battery-powered pulse generator implanted below the clavicle and a lead that is wrapped around the left vagus nerve in the carotid sheath [11]. Although complete seizure freedom with VNS insertion is rare, it is often beneficial in reducing seizure frequency and improving QOL [12].

Recently, many studies have reported that VNS therapy might be helpful to decrease seizure frequency. Gonzalez et al. [11] examined several studies assessing VNS efficacy and summarized that blinded randomized controlled trials for both children and adults with intractable epilepsy demonstrated that $23 \%$ to $57 \%$ of patients attain responder status (a seizure frequency reduction of at least 50\%) with short-term follow-up. In a multicenter study, DeGiorgio et al. [13] provided further evidence of VNS efficacy with the publication of a non-blinded randomized controlled trial of VNS implantation in 28 participants, resulting in a median seizure reduction of $30 \%$ and $45 \%$ of patients achieving responder status. From a systematic literature review Gonzalez et al. [11] also suggested that long-term studies have shown a progressive increase in response to VNS as duration of implantation increases. The data they examined, from 2,869 patients across 78 studies, showed an increase in both responder rate and seizure freedom rate over time [11]. Additionally, Kim and Kim [2] treated nine Korean LGS patients with VNS therapy and reported a mean reduction in seizure frequency of $52 \%$ after 6 months and $58 \%$ after 1 year.

Our study aimed to add further evidence of VNS efficacy in Korean patients, especially after a relatively long period of more than 24 months. Seizure frequency at the last follow-up decreased by $57.2 \%$ on average compared with the baseline and with non-parametric statistics a $P$-value of 0.028 was produced, which means that VNS resulted in a statistically significant decrease in seizure frequency. Examining the changes in seizure frequency in further detail, all the patients were responders directly after the VNS surgery. After several unstable months with significant fluctuations, the seizure frequency of most patients settled at a specific level and was subsequently maintained. 
At the last follow-up visit one patient was completely seizure free, but one patient was reported as having a similar seizure frequency compared to the baseline. The first child stopped having seizures 18 months after the VNS surgery and remained seizure free for 12 months. According to Braakman et al. [4], seizure freedom in LGS may rarely be achieved by VNS therapy and has been reported after a period of three years of VNS, which means our patient had a rapid response. In the study of Gonzalez et al. [11] 60\% of patients achieved responder status following VNS and only $8 \%$ of patients were seizure-free at the last follow-up. Therefore, it is necessary to monitor the patient's seizure frequency regularly for few more years, even if the child does not have any seizures, before making an accurate judgement about seizure-free status.

The second child with the similar seizure frequency compared to the baseline was a responder initially, directly after the VNS operation. The child's baseline seizure frequency was 15 which is relatively low and there were continuous fluctuations in seizure frequency after the VNS surgery. Just 6 months before his last follow-up visit the patient was a responder. With the initial baseline seizure frequency low, one can argue that smaller changes in frequency may be more significant for this patient than in a patient with a high baseline frequency. Thus, it might not always be that important to assess the exact reduction in monthly seizures, but to rather evaluate the patient's overall condition and subjective benefit, while monitoring changes in seizure frequency at regular intervals on a long-term basis.

VNS therapy has been reported to have a positive influence on QOL by several articles $[14,15]$. Clark et al. [16] suggested that the QOL in patients with over 50\% seizure reduction after VNS surgery were mainly due to the improvement of alertness, memory, and emotion. In our study clinician's utilized the CGI-I scale to assess the patient's overall condition, while the severity of illness and efficacy index scales were used to examine the outcomes of the VNS therapy. At the last follow-up assessment, there were four patients that scored "minimally improved," one patient "much improved," one patient "very much improved," and one patient "no change." A non-parametric test produced a $P$-value of 0.066 and as it is greater than 0.05 it means that there is no statistically significant improvement with VNS on the CGI-I scale. However, the $P$-value was close to 0.05 , so it appears that VNS tends to produce a positive effect on the CGI-I scale.

Several studies on the effects of VNS have also reported improvements of other factors related to QOL, including alertness, concentration, energy, memory, mood, verbal communication, progress with schoolwork and development of life skills [7]. Zamponi et al. [17] found that one-third of patients improve their adaptive behavior and half of patients reported a better QOL, de- spite epilepsy severity. Orosz et al. [7] showed an improvement in alertness in $66.1 \%$ of patients with adjunctive VNS and benefits in the areas of concentration, energy, mood, verbal communication and progress with school work in about one-third of patients at 24 months follow-up. Our study also gathered data relating to QOL from patients and caregivers at the outpatient visits. All the patients experienced improvement in alertness and there was one patient with a gradual reduction in drooling. Furthermore, one patient became potty-trained after VNS surgery which positively affect not only the quality of the patient's life, but also that of the caregiver's. One caregiver of another patient also expressed satisfaction about VNS therapy, reporting that the patient who was in a wheelchair at the first visit of the clinic became to be able to walk alone and have better communication skills with improvement of alertness. As mentioned like this, there were subjective descriptions from caregivers representing diverse range of symptom relieves and better conditions of the LGS patients. The current study did not set specific parameters relating to $\mathrm{QOL}$, but by providing the caregiver or patient with a survey that includes common elements of QOL, such as alertness, global interaction, or night-time sleep, more objective and accurate data can be obtained, particularly over a longer time period.

By anchoring and stimulating the vagus nerve, the VNS system lets patients control over-excitabilities without damaging brain tissue, and depending on the symptoms, modulate stimulation intensity [2]. Despite these advantages, there are complications of VNS therapy which can be classified into two categories: (1) those associated with surgical implantation and (2) those related to electrical stimulation [18]. According to Giordano et al. [19], the complications related to surgery include intraoperative bradycardia and asystole during lead impedance testing, hematoma, infections, and vagus nerve injury resulting in hoarseness, dyspnea or dysphagia due to left vocal cord paralysis. However, in most cases the paralysis only persists for a few months and then resolves [19]. As mentioned, patients can also suffer from complications associated with electrical stimulation of the vagus nerve. Examining several studies on the complications of stimulation, Gonzalez et al. [11] concluded that hoarseness is the most prevalent adverse effect and some studies have recently suggested an association between VNS and sleep apnea. In our study, there was only one patient who was admitted to the ICU due to pneumonia which, as an infection following the operation, can be classified as a complication of the VNS surgery. Apart from this case, there were no other complications related to the VNS surgeries and no symptoms associated with stimulation such as hoarseness, stimulation of the phrenic nerve due to proximity or obstructive sleep apnea.

There were four retrospective studies on VNS efficacy pub- 
lished in South Korea. Thus, the present article briefly reviewed those studies with Table 4 and looked into similarity or difference comparing to the current study. Kim et al. [20] retrospectively reviewed medical records of 12 patients (nine of them were LGS patients) with intractable children epilepsy who got VNS insertion from two university hospitals, and $67 \%$ of the whole patients got over 50\% reduction of seizure frequency. Among those patients, one patient with partial seizure showed a $90 \%$ reduction, and another patient originating from previous encephalitis got a $75 \%$ reduction at 12 and 24 months. Also, there were two patients with some improvements on EEG. Hoarseness, respiratory difficulty during sleep, infection of the surgical wound, increased salivation, and failure of pulse generator were transiently presented as the complications of VNS, and relieved after coordinating output current of the generator. You et al. [21] also retrospectively reviewed medical records of seven patients (two of them were LGS patients) with intractable children epilepsy who got VNS insertion from one medical center, and $71 \%$ of the whole patients showed greater than $50 \%$ reduction of seizure frequency. They indicated that the seizure reduction appeared 3 months after VNS insertion, and mentioned positive effects on EEG and QOL. The study also reported VNS complications such as hoarseness and wound infection appeared in few cases for short period.

Kang et al. [22] also retrospectively studied medical records on 297 patients with intractable children epilepsy, which is relatively big number compared with other studies from single medical center. They investigated the effects of antiepileptic drugs, prednisolone, ketogenic diet, epilepsy surgery, and VNS. Among the whole study population, five patients got VNS insertion and two of them were diagnosed with LGS. Over 50\% reduction of seizure frequency was appeared at $40 \%$ of those five patients, and the study concluded that VNS therapy appears to be successful regardless of seizure type or cause and attractive as non-pharmacologic aspects. Lastly, Yum et al. [23] retrospectively checked medical records on 79 LGS patients at one medical center and there were seven patients who got VNS therapy among the whole study population. Greater than 50\% reduction of seizure frequency were shown at $29 \%$ among those seven patients. Due to errors of pulse generator, one patient appears to have severe symptoms of stimulated vagus nerve, but continue the state of seizure free for several weeks at the same time. The study claimed that the number of patients with VNS therapy was too small to clearly figure out the efficacy of VNS, but there was no critical complication.

Although there were some differences between those four studies and the present study, the conclusion that VNS appears to be effective and safe choice for VNS was common. Also, the four studies and the current study similarly examined the efficacy of
VNS with seizure frequency reduction and mentioned complications of VNS therapy. Unlike the four articles which had relatively short minimum follow-up period or checked the seizure frequency for every 12 months, the present article indicated seizure frequency of each patient more specifically on Fig. 1, for every 6 months during relatively long follow-up period with minimum of 24 months. Looking through the Fig. 1, readers would be able to see the overall flow of seizure frequency on each patient. Also, You and colleagues mentioned the effects of VNS on QOL of the patients only with the reports from caregiver about few factors related to QOL such as alertness, communication or exercise skills. On the other hand, the present article described QOL with both CGI-I scale and reports from caregivers of the patients, differed from the other studies.

The present study has a few potential limitations. The study was based on the data of only seven patients at a single medical center. It would be of great help to recruit additional patients by cooperating with other hospitals to draw more universal conclusions and identify various etiologies. Although all the follow-up periods of seven patients was more than 24 months, performing consistent follow-up for each patient would also enable the collection of broader data. The other limitation is that our study mainly utilized caregivers' reports to assess changes in seizure frequency. Considering the age of the patients and specificity of current equipment available used to monitor for seizures such as generalized tonic-clonic seizures, using a device like an epilepsy-recording bracelet might be helpful to gather more objective and accurate data.

Our study established that VNS therapy positively affects LGS patients in terms of decreasing seizure frequency and improving QOL. Apart from rare complications from the VNS surgery and vagal stimulation, VNS can be regarded as a relatively safe and effective treatment modality for patients with LGS, which is a very challenging epileptic encephalopathy to manage. In future, more multicenter studies with more objective data collection conducted over longer time periods are necessary for further evidence of VNS use in LGS. As mentioned before, most studies about VNS including the current study examine the medical records retrospectively. For establishing VNS as a safe and reliable option to pharmaco-resistant epileptic patients, it would be helpful to follow-up on patients who received VNS therapy decades ago in their childhood, and prospectively identify the current efficacy plus any late complications.

\section{Conflicts of interest}

No potential conflicts of interest relevant to this article was reported. 


\section{Acknowledgements}

This work was supported by the research fund of Chungnam National University (2018-1299-01). This work was presented at the Korean Pediatric Society Congress in 2017.

\section{ORCID}

Bu Seon Kang, https://orcid.org/0000-0001-5787-6175

Joon Won Kang, https://orcid.org/0000-0001-5756-3814

\section{References}

1. Dupont S, Banica-Wolters R, An-Gourfinkel I, Lambrecq V, Navarro V, Adam C, et al. Understanding Lennox-Gastaut syndrome: insights from focal epilepsy patients with Lennox-Gastaut features. J Neurol 2017;264:1388-96.

2. Kim DS, Kim CS. Vagus nerve stimulation treatment for children with refractory epilepsy (Lennox-Gastaut syndrome). Korean J Clin Lab Sci 2007;39:56-62.

3. Rathaur BP, Garg RK, Malhotra HS, Kumar N, Sharma PK, Verma R, et al. Lennox-Gastaut syndrome: a prospective follow-up study.J Neurosci Rural Pract 2017;8:225-7.

4. Braakman HM, Creemers J, Hilkman DM, Klinkenberg S, Koudijs SM, Debeij-van Hall M, et al. Improved seizure control and regaining cognitive milestones after vagus nerve stimulation revision surgery in Lennox-Gastaut syndrome. Epilepsy Behav Case Rep 2018;10:111-3.

5. Katagiri M, Iida K, Kagawa K, Hashizume A, Ishikawa N, Hanaya $\mathrm{R}$, et al. Combined surgical intervention with vagus nerve stimulation following corpus callosotomy in patients with Lennox-Gastaut syndrome. Acta Neurochir (Wien) 2016;158:1005-12.

6. Shahwan A, Bailey C, Maxiner W, Harvey AS. Vagus nerve stimulation for refractory epilepsy in children: more to VNS than seizure frequency reduction. Epilepsia 2009;50:1220-8.

7. Orosz I, McCormick D, Zamponi N, Varadkar S, Feucht M, Parain D, et al. Vagus nerve stimulation for drug-resistant epilepsy: a European long-term study up to 24 months in 347 children. Epilepsia 2014;55:1576-84.

8. You SJ, Kang HC, Ko TS, Kim HD, Yum MS, Hwang YS, et al. Comparison of corpus callosotomy and vagus nerve stimulation in children with Lennox-Gastaut syndrome. Brain Dev 2008;30:195-9.

9. Engel J, Pedley T, Aicardi J, Dichter MA, Moshe S. Epilepsy: a comprehensive textbook. 2nd ed. Vol 3. Philadelphia: Lippincott Williams \& Wikins; 2008.
10. VanStraten AF, Ng YT. Update on the management of Lennox-Gastaut syndrome. Pediatr Neurol 2012;47:153-61.

11. Gonzalez HFJ, Yengo-Kahn A, Englot DJ. Vagus nerve stimulation for the treatment of epilepsy. Neurosurg Clin N Am 2019; 30:219-30.

12. Englot DJ. A modern epilepsy surgery treatment algorithm: incorporating traditional and emerging technologies. Epilepsy Behav 2018;80:68-74.

13. DeGiorgio C, Heck C, Bunch S, Britton J, Green P, Lancman M, et al. Vagus nerve stimulation for epilepsy: randomized comparison of three stimulation paradigms. Neurology 2005;65:317-9.

14. Ulate-Campos A, Cean-Cabrera L, Petanas-Argemi J, Garcia-Fructuoso G, Aparicio J, Lopez-Sala A, et al. Vagus nerve stimulator implantation for epilepsy in a paediatric hospital: outcomes and effect on quality of life. Neurologia 2015;30:465-71.

15. Hauptman JS, Mathern GW. Vagal nerve stimulation for pharmacoresistant epilepsy in children. Surg Neurol Int 2012;3 (Suppl 4):S269-74.

16. Clark KB, Naritoku DK, Smith DC, Browning RA, Jensen RA. Enhanced recognition memory following vagus nerve stimulation in human subjects. Nat Neurosci 1999;2:94-8.

17. Zamponi N, Passamonti C, Cesaroni E, Trignani R, Rychlicki F. Effectiveness of vagal nerve stimulation (VNS) in patients with drop-attacks and different epileptic syndromes. Seizure 2011; 20:468-74.

18. George MS, Aston-Jones G. Noninvasive techniques for probing neurocircuitry and treating illness: vagus nerve stimulation (VNS), transcranial magnetic stimulation (TMS) and transcranial direct current stimulation (tDCS). Neuropsychopharmacology 2010;35:301-16.

19. Giordano F, Zicca A, Barba C, Guerrini R, Genitori L. Vagus nerve stimulation: Surgical technique of implantation and revision and related morbidity. Epilepsia 2017;58 Suppl 1:85-90.

20. Kim JT, Kim YH, Kang HC, Eun S, Hwang YS, Kim DS, Kim HD. Therapeutic outcomes of vagus nerve stimulation in intractable childhood epilepsy. J Korean Child Neurol Soc 2004;12:132-43.

21. You SJ, Kim DS, Lee JK, Ko TS. Vagus nerve stimulation in intractable pediatric epilepsy patients. J Korean Child Neurol Soc 2005; 13:8-14.

22. Kang HC, Kwon JW, Hwang YS, Kim HD, Park SK. Outcomes of therapeutic modalities for intractable childhood epilepsy. J Korean Child Neurol Soc 2005; 13:152-64.

23. Yum MS, Hong SH, Lee JK, You SJ, Kim DS, Ko TS. Multimodal management of Lennox-Gastaut syndrome. J Korean Child Neurol Soc 2007;15:154-61. 